\title{
INFLUENCE OF THE MAGNETIC FIELD ON THE LATTICE DYNAMICS OF AN IONIC CRYSTAL IN THE LONG-WAVE LIMIT
}

T. ORD. MAGNETVALJA MOJU IOONKRISTALlivORE DUNAAMIKALE PIKALAINELISEL PIIRJUHUL

T. ЭРД. ВЛИЯНИЕ МАГНИТНОГО ПОЛЯ НА ДИНАМИКУ РЕШЕТКИ ИОННОГО КРИСТАЛЛА В ДЛИННОВОЛНОВОМ ПРЕДЕЛЕ

\section{(Presented by V. Hizhnyakov)} $\vec{B})$ on the long-wave lattice vibrations of a cubic ionic crystal have been

In this report, the influence of a homogeneous magnetic field (induction considered in the continuous approximation with the allowance made for the splitting of the unperturbed frequencies into longitudinal and transversal components. The general formalism for describing the ionic crystal vibrations in the presence of the magnetic field has been developed In [ $\left.{ }^{1}\right]$, where a concrete two-dimensional lattice model has also been studied. Earlier, in $\left[{ }^{2}\right]$, some aspects of the problem were analysed, proceeding from symmetry considerations.

Let the unit cell of a crystal consist of two ions with effective charges $\pm \bar{e}$ and masses $m_{ \pm}$. The equations of motion for long-wave lattice vibrations with $\overrightarrow{\mathrm{q}}=0$ in the continuous approximation [ [ $\left.{ }^{3}\right]$ for $\overrightarrow{B \neq 0}$ have the form

$$
\begin{gathered}
\ddot{w}_{j}=-\omega_{j}^{2} w_{j}+\sum_{j^{\prime} j^{\prime \prime}} \varepsilon_{j j^{\prime} j^{\prime \prime}}\left[\omega_{c 1} \dot{w}_{j^{\prime}}+\omega_{c 2} \dot{c}_{j^{\prime}}\right] b_{j^{\prime \prime}} ; \\
\ddot{c}_{j}=\omega_{c 3} \sum_{j^{\prime} j^{\prime \prime}} \varepsilon_{j j^{\prime} j^{\prime \prime}} \dot{w}_{j^{\prime}} b_{j^{\prime \prime}}, \quad j=1,2,3 .
\end{gathered}
$$

Here

$$
\begin{gathered}
\omega_{1,2}=\omega_{t}, \quad \omega_{3}=\omega_{l}, \\
\overrightarrow{\mathrm{w}}=\overrightarrow{\mathrm{u}}_{+}-\overrightarrow{\mathrm{u}}_{-}, \quad \overrightarrow{\mathrm{c}}=\frac{\overrightarrow{\mathrm{u}_{+}} m_{+}+\overrightarrow{\mathrm{u}}_{-} m_{-}}{m_{+}+m_{-}}, \quad \overrightarrow{\mathrm{b}}=\overrightarrow{\mathrm{B}} / B, \\
\omega_{c \mu}=\bar{e} B / M_{\mu}, \quad M_{1,2}^{-1}=m_{+}^{-1} \mp m_{-}^{-1}, \quad M_{3}=m_{+}+m_{-} ;
\end{gathered}
$$

$\overrightarrow{\mathrm{u}}_{ \pm}$are the shifts of positive and negative ions; $\omega_{t, l}$ are the limiting frequencies of transversal and longitudinal optic vibrations; $\varepsilon_{j j^{\prime} j^{\prime \prime}}$ are the components of the Levi-Civita tensor. Vibrational motion of the centre 
of gravity of ions, described by Eqs (2), appears due to the interaction of optic modes with the magnetic field.

Assuming

$$
w_{j}=a_{j} \exp (\tilde{i \tilde{\omega} t}), \quad c_{j}=\alpha_{j} \exp (\tilde{i \tilde{\omega} t}) ;
$$

the system of differential equations (1), (2) leads to

$$
\begin{gathered}
\sum_{j^{\prime}}\left\{\left[\tilde{\omega}^{2}-\omega_{j}^{2}\right] \delta_{j j^{\prime}}+i \omega_{c 1} \tilde{\omega} \sum_{j^{\prime \prime}} \varepsilon_{j j^{\prime} j^{\prime \prime}} b_{j^{\prime \prime}}-\omega_{c 2} \omega_{c 3}\left[\delta_{j j^{\prime}}-b_{j} b_{j^{\prime}}\right]\right\} a_{j^{\prime}}=0, \\
-\tilde{\omega} \alpha_{j}=i \omega_{c 3} \sum_{j^{\prime} j^{\prime \prime}} \varepsilon_{j j^{\prime} j^{\prime \prime}} a_{j^{\prime}} b_{j^{\prime \prime}} .
\end{gathered}
$$

For $\omega_{\mathrm{c} 1} / \omega_{t} \ll 1, \quad \omega_{\mathrm{c} 1} / \sqrt{\omega_{l}^{2}-\omega_{t}^{2}} \ll 1, \sqrt{\omega_{\mathrm{c} 2} \omega_{\mathrm{c} 3} / \omega_{t, l}} \ll 1$ from the condition of the existence of a nontrivial solution for the system of equations (7) by the iterative method we find for the eigenfrequencies in the first approximation of the magnetic field*

$$
\begin{gathered}
\tilde{\omega}_{1,2}^{2}=\omega_{t}^{2} \pm \omega_{t}\left|\omega_{c 1} \cos \varphi\right|, \\
\tilde{\omega}_{3}^{2}=\omega_{l}^{2},
\end{gathered}
$$

and in the second approximation

$$
\begin{gathered}
\tilde{\omega}_{1,2}^{2}=\omega_{t}^{2}-\frac{1}{2}\left[\frac{\omega_{c 1}^{2} \omega_{t}^{2} \sin ^{2} \varphi}{\omega_{l}^{2}-\omega_{t}^{2}}-\omega_{c 2} \omega_{c 3}\left(1+\cos ^{2} \varphi\right)\right] \pm \\
\pm \frac{1}{2}\left\{\left[\frac{\omega_{c 1}^{2} \omega_{t}^{2}}{\omega_{l}^{2}-\omega_{t}^{2}}-\omega_{c 2} \omega_{c 3}\right]^{2} \sin ^{4} \varphi+4 \omega_{c 1}^{2}\left[\omega_{t} \pm \frac{1}{2}\left|\omega_{c 1} \cos \varphi\right|\right]^{2} \cos ^{2} \varphi\right\}^{1 / 2} \\
\tilde{\omega}_{3}^{2}=\omega_{l}^{2}+\left[\frac{\omega_{c 1}^{2} \omega_{l}^{2}}{\omega_{l}^{2}-\omega_{t}^{2}}+\omega_{c 2} \omega_{c 3}\right] \sin ^{2} \varphi
\end{gathered}
$$

where

$$
\cos \varphi=b_{3}=\vec{b} \cdot \vec{o}, \quad \vec{o}=\frac{\vec{q}}{q}, \quad 0 \leqslant \varphi \leqslant \pi / 2 .
$$

On the basis of Eqs (2) and (6) it follows that

$$
\tilde{\omega}_{4,5,6}=0 \text {. }
$$

According to Eqs (7)-(12), (14) the vibrational motions of the centre of gravity of the unit cell, induced by the magnetic field, occur on optic frequencies. It means that the crystal serves as a hypersound generator for $B \neq 0$.

It can be seen from Eqs (9)-(12) that the magnetic field mixes the transversal optic vibrations in the first order of $B$ already, the transversal and longitudinal optic vibrations, however, do it in the second order. The induced motion of the centre of gravity of the unit cell gives a contribution to $\tilde{\omega}_{1,2,3}$ in the second order of the magnetic field. Eigenfrequencies $\tilde{\omega}_{1,2,3}$ form one-dimensional bands in the $\varphi$-space, as the angle

* In the cases $b_{3}=0$ and $b_{3}= \pm 1$ the problem has a simple precise solution. 
$\varphi$ passes through all the values in the interval from 0 to $\pi / 2$. The dependence $\tilde{\omega}_{1,2,3}$ on $\varphi$ is caused by the unequality of the frequencies $\omega_{l}$ and $\omega_{t}$.

Now; on the basis of Eqs (9)-(12) we estimate the shifts of the eigenfrequencies $\left.D_{j}=\tilde{\omega}_{j}-\omega_{j}\right)$ for $\mathrm{LiH}\left(\omega_{l}=2.1 \cdot 10^{14} \mathrm{~s}^{-1}, \omega_{t}=1.1 \cdot 10^{14} \mathrm{~s}^{-1}\right.$ $\left.\left[{ }^{4}\right] ; \mathrm{m}_{+}=1.15 \cdot 10^{-23} \mathrm{~g}, \mathrm{~m}_{-}=0.17 \cdot 10^{-23} \mathrm{~g}, \bar{e}=e\right)$. For $B=20 \mathrm{~T}$ the maximum values of $D_{j}$ equal: $D_{1,2}(\varphi=0) \approx \pm 8 \cdot 10^{8} \mathrm{~s}^{-1} ; D_{3}(\varphi=\pi / 2) \approx 10^{4} \mathrm{~s}^{-1}$. Such value of $D_{3}$ is negligible, but in agreement with [1], the shifts $D_{1,2}$ should be experimentally observable.

The author wishes to thank N. Kristoffel, P. Konsin, and A. Pishchev for discussions.

\section{REFERENCES}

1. Vineyard, G. H. Phys. Rev. B, 31, № 2, 814-820 (1985).

2. Anastasskis, E., Burstein, E., Maradudin, A. A., Minnick, R. J. Phys. Chem. Solids, 33, № 2, 519-531 (1972).

3. Борн М., Кунь Х. Динамическая теория кристаллических решеток. М., ИЛ, 1958.

4. Киттель Ч. Введение в физику твердого тела. М., «Наука», 1978.

Academy of Sciences of the Estonian SSR,

Institute of Physics

Received Apr. 23, 1986 